\title{
A NOTE ON UNIQUE CONTINUATION FOR SCHRÖDINGER'S OPERATOR
}

\author{
CARLOS E. KENIG AND CHRISTOPHER D. SOGGE \\ (Communicated by Walter Littman)
}

\begin{abstract}
In this paper we shall prove a unique continuation theorem for Schrödinger's operator, $i \partial / \partial t-\Delta$. This will be a consequence of "uniform Sobolev inequalities" for operators which are the Schrödinger operator plus lower order terms in $x$.
\end{abstract}

In this paper, we shall prove unique continuation theorems for solutions to certain differential inequalities involving the Schrödinger operator on $\mathbf{R}^{n+1}$,

$$
i \frac{\partial}{\partial t}+\Delta \text {. }
$$

Here, $\Delta$ denotes the Laplace operator on $\mathbf{R}^{n}$. We shall show that, if $n \geq 1$, and if $u(x, t)$ satisfies certain global integrability conditions as well as a differential inequality $\left|\left(i \frac{\partial}{\partial t}+\Delta\right) u\right| \leq|V u|$, where $V(x, t) \in L^{n+2 / 2}\left(\mathbf{R}^{n+1}\right)$, then $u$ must vanish identically if it vanishes in a halfspace.

These results should be compared to similar results for the wave operator obtained in [3]. As in that work, a key ingredient in the proof will be a restriction theorem for the Fourier transform. For the Schrödinger operator, we will want to make use of the following restriction lemma of Strichartz [5].

LEMMA 1. Let $\hat{f}(\xi, \tau)$ denote the $(n+1)$-dimensional Fourier transform of $f(x, t)$. Then, if $n \geq 1$ and if $p=2(n+2) /(n+4)$, (i.e. $\frac{1}{p}-\frac{1}{p^{\prime}}=\frac{2}{n+2}$, where $p^{\prime}$ denotes the dual exponent), one has

$$
\left\|\int_{\mathbf{R}^{n}} \hat{f}\left(\xi,|\xi|^{2}\right) e^{i\left\langle(x, t),\left(\xi,|\xi|^{2}\right)\right\rangle} d \xi\right\|_{L^{p^{\prime}\left(\mathbf{R}^{n+1}\right)}} \leq C\|f\|_{L^{p}\left(\mathbf{R}^{n+1}\right)} .
$$

In order to state our main result, let us let $L(D)$ denote an arbitrary constant coefficient operator in $x$ :

$$
L(D)=\left\langle a, \nabla_{x}\right\rangle+b,
$$

where $a$ and $b$ are complex. Then, if from now on, we let $p$ and $p^{\prime}$ be the exponents defined in the lemma, we shall prove the following 'uniform Sobolev inequality' for the Schrödinger operator which generalizes a result of Strichartz [4] for the special case where $L(D)=0$.

Received by the editors May 14, 1987.

1980 Mathematics Subject Classification (1985 Revision). Primary 35L15, 42B25.

Key words and phrases. Sobolev inequalities, unique continuation, restriction theorems.

Supported in part by an NSF grant and a Guggenheim fellowship.

Supported by an NSF postdoctoral fellowship. This research was in part carried out at the I.H.E.S., whom this author would like to thank. 
THEOREM 1. If $n \geq 1$, then there exists a constant $C$, independent of $L(D)$, so that

(2) $\|u(x, t)\|_{L^{p^{\prime}}\left(\mathbf{R}^{n+1}\right)} \leq C\left\|\left(i \frac{\partial}{\partial t}+\Delta+L(D)\right) u(x, t)\right\|_{L^{p}\left(\mathbf{R}^{n+1}\right)}$

$$
u \in W^{p}\left(\mathbf{R}^{n+1}\right) .
$$

Here, the "Sobolev space," $W^{p}$, is defined to be the functions $u(x, t)$ with the property that $\left[\left(\sqrt{\left(1+\tau^{2}\right)}+|\xi|^{2}\right) \hat{u}(\xi, \tau)\right]^{\vee} \in L^{p}\left(\mathbf{R}^{n+1}\right)$. This of course insures that the functions in the right-hand side of $(2)$ are in $L^{p}\left(\mathbf{R}^{n+1}\right)$.

Next, we claim that an immediate corollary of the uniform inequalities (2) is the following Carleman inequality valid for arbitrary $\lambda \in \mathbf{R}, \nu \in \mathbf{R}^{n+1}$,

$$
\left\|e^{\lambda\langle(x, t), \nu\rangle} u(x, t)\right\|_{L^{p^{\prime}}\left(\mathbf{R}^{n+1}\right)} \leq C\left\|e^{\lambda\langle(x, t), \nu\rangle}\left(i \frac{\partial}{\partial t}+\Delta\right) u(x, t)\right\|_{L^{p}\left(\mathbf{R}^{n+1}\right)},
$$

That (3) is a consequence of (2) just follows from the fact that given $\lambda \in \mathbf{R}$, $\nu \in \mathbf{R}^{n+1}$, there is an $L(D)$ as above so that

$$
e^{\lambda\langle(x, t), \nu\rangle}\left(i \frac{\partial}{\partial t}+\Delta\right) e^{-\lambda\langle(x, t), \nu\rangle}=i \frac{\partial}{\partial t}+\Delta+L(D) .
$$

Furthermore, since well-known arguments show that Carleman inequalities imply unique continuation theorems, the following result is a corollary of (3). Since similar proofs are given in [1], [3], we shall not give the proof of the corollary.

COROLlaRY 1. Suppose that $u(x, t) \in W^{p}\left(\mathbf{R}^{n+1}\right)$ and that $u(x, t)$ satisfies the differential inequality

$$
\left|\left(i \frac{\partial}{\partial t}+\Delta\right) u(x, t)\right| \leq|V(x, t) u(x, t)|
$$

for some potential $V \in L^{n+2 / 2}\left(\mathbf{R}^{n+1}\right)$. Then if $u$ vanishes in some half space of $\mathbf{R}^{n+1}$, it follows that $u$ vanishes identically.

Let us now turn to the proof of Theorem 1. We will need to make use of Lemma 1 and the following special case of $(2)$ where $L(D)=z, z \in \mathbf{C}$.

LEMMA 2. There is a constant $C$ so that for all $u \in W^{p}$ and $z \in \mathbf{C}$,

$$
\|u(x, t)\|_{L^{P^{\prime}\left(\mathbf{R}^{n+1}\right)}} \leq C\left\|\left(i \frac{\partial}{\partial t}+\Delta+z\right) u(x, t)\right\|_{L^{p}\left(\mathbf{R}^{n+1}\right)} .
$$

Let us for now assume this result and show how it implies Theorem 1. First let us note that elementary arguments (cf. [3]) reduce the proof of (2) to the special cases where

$$
L(D)=\varepsilon \frac{\partial}{\partial x_{n}}+i \beta, \quad \varepsilon, \beta \in \mathbf{R} .
$$

For simplicity, we shall also assume that $\varepsilon=1$ and $\beta=0$ since the same type of arguments will handle the other cases. Consequently, we have reduced matters to verifying the following "multiplier inequality:"

$$
\left\|\left\{\frac{\hat{f}(\xi, \tau)}{\tau+|\xi|^{2}+i \xi_{n}}\right\}^{\vee}\right\|_{p^{\prime}} \leq C\|f\|_{p}, \quad f \in \mathscr{S} .
$$


Next, as in [3], since $p<2<p^{\prime}$, it follows easily from Littlewood-Paley theory that (4) would follow if we could show that there is a constant $C$ independent of $k \in \mathbf{Z}$ so that (4) holds whenever $f \in \mathscr{S}$ has the property that $\hat{f}(\xi, \tau)=0$, when $\left|\xi_{n}\right| \notin\left[2^{-k}, 2^{-k+1}\right]$. To deal with such functions, we shall use the following inequality which is a consequence of Lemma 2 .

$$
\left\|\left\{\frac{\hat{f}(\xi, \tau)}{\tau+|\xi|^{2}+i 2^{-k}}\right\}^{\vee}\right\|_{p^{\prime}} \leq C \|\left. f\right|_{p}, \quad f \in \mathscr{S} .
$$

Therefore, if $\hat{f}$ is supported as above, we need only prove that

$$
\left\|\left\{\frac{\left(2^{-k}-\xi_{n}\right) \hat{f}(\xi, \tau)}{\left(\tau+|\xi|^{2}+i \xi_{n}\right)\left(\tau+|\xi|^{2}+i 2^{-k}\right)}\right\}^{\vee}\right\|_{p^{\prime}} \leq C\|f\|_{p} .
$$

It is now easy to finish the proof. In fact if one makes the change of variables to parabolic coordinates, $(\xi, \tau) \rightarrow(\eta, \rho)=\left(\xi, \tau+|\xi|^{2}\right)$, it is easy to see that Strichartz's restriction inequality implies that the left-hand side of $(5)$ is dominated by

$$
\int_{-\infty}^{\infty}\left\|\left\{\frac{\left(2^{-k}-\xi_{n}\right) \hat{f}(\xi, \tau)}{\left(\rho+i \xi_{n}\right)\left(\rho+i 2^{-k}\right)}\right\}^{\vee}\right\|_{p} d \rho .
$$

But on the other hand, since we are assuming that $\hat{f}(\xi, \tau)=0$ unless $\left|\xi_{n}\right| \epsilon$ $\left[2^{-k}, 2^{-k+1}\right]$, it follows that the last expression is majorized by

$$
\int_{-\infty}^{\infty} \frac{2^{-k} d \rho}{\rho^{2}+2^{-2 k}}\|f\|_{p}=C\|f\|_{p}
$$

which of course finishes the proof of (5).

Finally, to finish things, we must prove Lemma 2. As above, straightforward arguments reduce things to the case where $z$ is purely imaginary. Further, by homogeneity, we can further reduce things to the case where $z=i$ and so we need only prove that

$$
\left\|\left\{\frac{\hat{f}(\xi, \tau)}{\tau+|\xi|^{2}+i}\right\}^{\vee}\right\|_{p^{\prime}} \leq C\|f\|_{p}
$$

Let $S: f \rightarrow S f(x, t)$ denote the operator in the left-hand side of (6). Then, by taking a partial Fourier transform in $\tau$, it follows that if $t \in \mathbf{R}$ is fixed and if $a(s)=\int_{-\infty}^{\infty}(\tau+i)^{-1} e^{i \tau s} d \tau$ then

$$
S f(x, t)=\int_{-\infty}^{\infty}\left(\int_{\mathbf{R}^{n}} \tilde{f}(\xi, t-s) e^{i s|\xi|^{2}} e^{i x \cdot \xi}\right) a(s) d s,
$$

where $\tilde{f}$ denotes the Fourier transforms in $x$. Note that $a(s)$ is a bounded function.

Next, let us recall that

$$
\left\|\int_{\mathbf{R}^{n}} \tilde{g}(\xi) e^{i s|\xi|^{2}} e^{i x \cdot \xi} d \xi\right\|_{L^{p^{\prime}\left(\mathbf{R}^{n}\right)}} \leq C|s|^{-n / 2\left(1 / p-1 / p^{\prime}\right)}\|g\|_{L^{p}\left(\mathbf{R}^{n}\right)} .
$$

To prove this inequality, one could first note that, by homogeneity, one can always assume that $s=1$. Then, since $e^{i|\xi|^{2}}$ is a bounded $L^{2}\left(\mathbf{R}^{n}\right) \rightarrow L^{2}\left(\mathbf{R}^{n}\right)$ 
and $L^{1}\left(\mathbf{R}^{n}\right) \rightarrow L^{\infty}\left(\mathbf{R}^{n}\right)$ multiplier, (8) follows from an application of the M. Riesz interpolation theorem.

To finish, let us note that Minkowski's integral inequalities (7) and (8) imply that for fixed $t \in \mathbf{R}$,

$$
\begin{aligned}
\|S f(\cdot, t)\|_{L^{p^{\prime}}\left(\mathbf{R}^{n}\right)} & \leq C \int_{-\infty}^{\infty}\left\|\int_{\mathbf{R}^{n}} \tilde{f}(\xi, t-s) e^{i s|\xi|^{2}} e^{i x \cdot \xi} d \xi\right\|_{L^{p^{\prime}}\left(\mathbf{R}^{n}\right)} d s \\
& \leq C \int_{-\infty}^{\infty}\|f(\cdot, t-s)\|_{L^{p}\left(\mathbf{R}^{n}\right)}|s|^{-n / 2\left(1 / p-1 / p^{\prime}\right)} d s .
\end{aligned}
$$

Finally, since an easy computation shows that $1-\left(1 / p-1 / p^{\prime}\right)=n / 2\left(1 / p-1 / p^{\prime}\right)$, (6) follows from this last inequality if one now uses fractional integration in $\mathbf{R}$.

\section{REFERENCES}

1. L. Hörmander, Uniqueness theorems for second-order elliptic differential operators, Comm. Partial Differential Equations 8 (1983), 21-64.

2. D. Jerison and C. E. Kenig, Unique continuation and absence of positive eigenvalues for Schrödinger operators, Ann. of Math. 121 (1985), 463-494.

3. C. E. Kenig, A. Ruiz, and C. D. Sogge, Uniform Sobolev inequalities and unique continuation theorems for second-order constant coefficient differential operators, Duke Math. J. 55 (1987), 329-347.

4. R. S. Strichartz, A priori estimates for the wave equation and some applications, J. Funct. Anal. 5 (1970), 218-235.

5. $\frac{}{714}$, Restrictions of Fourier transforms to quadratic surfaces, Duke Math. J. 44 (1977), 705714.

Department of Mathematics University of Chicago, Chicago, Illinois 60637 